\title{
Temperament and behaviour problems in young children with Down syndrome at 12,30 , and 45 months
}

\author{
Deborah J. Fidler', David E. Most ${ }^{2}$, Cathryn Booth-LaForce ${ }^{3} \&$ Jean F. Kelly \\ ' Human Development and Family Studies, Colorado State University, \\ ${ }^{2}$ School Of Education, Colorado State University, ${ }^{3}$ Family \& Child Nursing, University of Washington
}

\begin{abstract}
Though cross-sectional studies have yielded important information regarding the trajectory of psychopathology in middle childhood and adolescence in Down syndrome, there has been little exploration of maladaptive behaviour in the earliest years of development. In this study, we explore the emergence of maladaptive behaviour in young children with Down syndrome ( $n$ $=24)$ and a mental age-matched comparison group $(n=33)$ of young children with developmental disabilities of mixed etiologies. Behavioural data (Bayley Scales of Infant Development, Infant Temperament Questionnaire, Achenbach's Child Behaviour Checklist) were collected for children in each group at 12 months, 30 months, and 45 months. Findings from this study suggest that the onset of internalising behaviour difficulties in young children with Down syndrome may emerge later in early development than in children with developmental disabilities of mixed/nonspecific etiologies. In addition, temperament at Time I appeared to be a much stronger predictor of maladaptive behaviour outcomes at Time 3 in the mixed comparison group than in the Down syndrome group. Implications of this trajectory of the early development of maladaptive behaviour in Down syndrome for intervention for young children with Down syndrome are discussed.
\end{abstract}

Keywords: maladaptive behaviour, Down syndrome, early development, behavioural phenotype

Previous comparison studies have reported that individuals with Down syndrome show lower levels of psychopathology than do other individuals with developmental disabilities (Dykens \& Kasari, 1997; Lund, 1985; Myers \& Pueschel, 1991; Stores et al. 1998). Despite these lower rates of psychopathology, individuals with Down syndrome show higher rates of behaviour problems than do typically developing children, and they are also at heightened risk for certain comorbid psychiatric diagnoses, such as autism or conduct disorder (Collacott et al. 1992; Kent et al. 1999; Lund, 1988). Identifying and understanding the manifestation of maladaptive behaviour, or problem behaviours that interfere with a child's adaptation, in Down syndrome may be especially important for service providers and caregivers, who may fall prey to the phenomenon of "diagnostic overshadowing" - attributing all atypicality in an individual with a genetic disorder to the multi-system disorder that has already been diagnosed. For individuals who do show maladaptive behaviour and psychopathology in addition to Down syndrome, specific intervention and therapeutic techniques may be a crucial aspect of an effective service plan.

While few population-based prevalence studies have been conducted, findings to date suggest that individuals with Down syndrome may be at risk for specific types of behaviour problems. For example, Collacott et al., (1992) reported higher rates of comorbid depression and dementia in adults with Down syndrome compared to a group of adults with developmental disabilities of mixed etiologies. In contrast, higher rates of other disorders, including conduct disorder, personality disorder, and schizophrenia, were reported in the mixed comparison group.

Yet these cross-sectional studies ignore the developmental changes that may be associated with the manifestation of behavioural problems in Down syndrome throughout the lifespan. Particular stages in development may be associated with a risk for specific types of behaviour problems, but not others. For example, Myers and Pueschel (1991) report that Disruptive Disorders was the most common category of psychiatric diagnosis in individuals with Down 
syndrome under age 20. With, $6.1 \%$ meeting criteria for Attention Deficit Disorder, $5.4 \%$ meeting criteria for Conduct/Oppositional disorder, and $6.5 \%$ meeting criteria for Aggressive behaviour, this category of behaviour problems was far more common than anxiety disorders and repetitive behaviours. In contrast, the most common diagnoses in their sample of adults with Down syndrome (over the age of 20$)$, were major-depressive disorders $(6.1 \%)$ and aggressive behaviour $(6.1 \%)$.

Dykens and colleagues (2002) also report increases in maladaptive behaviour with age in a sample of 4-19 year old individuals with Down syndrome on Achenbach's Child Behaviour Checklist (CBCL; Dykens et al., 2002). The CBCL generates information relating to total behaviour problems, as well as specific syndromes of internalising or externalising behaviour. Internalising behaviours fall under the anxiety, depression, and withdrawn categories; externalising behaviours refer fall under the categories of aggression or destruction. In the Dykens et al. (2002) study, 10-19 year old children with Down syndrome showed more internalising behaviours than the 4-6 year old children in their sample, and 10-13 year old children showed more externalising behaviours than the 4-6 year old group. Findings from this study also suggest that specific behavioural problems are already present in young children with Down syndrome ages 4 to 6 years. Specifically, $71 \%$ of $4-6$ year old children had parents who endorsed the description "cannot concentrate", $61 \%$ were rated as "demands attention", $51 \%$ received ratings for "argues a lot", and $28 \%$ received ratings for "prefers to be alone".

Though these studies have yielded important information regarding the trajectory of psychopathology in Down syndrome, there has been little exploration of maladaptive behaviour in the earliest years of development. In addition, there are no existing longitudinal studies of changes in maladaptive behaviour profiles in early childhood, or at any other time in development. Yet, lifespan findings from developmental psychopathology research suggest that there are continuities in developmental pathways in the area of maladaptive behaviour (Robins, 1966; 1978; Robins \& Rutter, 1990). It may be important to describe the earliest origins of maladaptive behaviour in this population longitudinally in order to craft targeted interventions that are informed by an understanding of developmental influences on maladaptive behaviour.

How early do signs of maladaptive behaviour become apparent in this population? Is temperament in infancy a predictor of maladaptive behaviour profiles in toddlerhood and the preschool years? Do individuals with Down syndrome differ from other children with developmental delays in the manifestation of these behaviour problems? Understanding the emergence of maladaptive behaviours in young children with Down syndrome may shed light on the origins of later comorbid psychiatric diagnoses individuals with Down syndrome, and may also offer important information regarding early intervention and family supports (Fidler, 2005).

In this study, we explore the emergence of maladaptive behaviour in young children with Down syndrome and a comparison group of young children with developmental disabilities of mixed etiologies. Behavioural data (Bayley Scales of Infant Development, Infant Temperament Questionnaire, Achenbach's Child Behaviour Checklist) were collected for children in each group at 12 months, 30 months, and 45 months. Between-group differences in behavioural functioning will be explored at each time point. In addition, the association between temperament and maladaptive behaviour will be explored longitudinally.

\section{Method}

\section{Participants}

Participants were 57 children with developmental delays and their parents (Down syndrome $n=24$; nonspecific developmental delays, $n=33$ ). For the Down syndrome group, all participants had a genetic diagnosis of trisomy 21 . For the comparison group, all children had idiopathic (nonspecific) developmental delays; no child in this group had any identifiable cause for their developmental delays. All children in the comparison group showed delays that qualified them for early intervention services in their local communities, though the specific criteria used for qualifying for services varied among the communities.

Families of children in this study in both groups were predominantly Caucasian. The majority of mothers in each group were married at Time 1 . All child participants were currently living at home. However, the two groups did differ in three important ways. Families of children with Down syndrome had approximately $\$ 20,000$ higher mean incomes, mothers of children with Down syndrome were approximately 3.7 years older than were mothers of children in the comparison group, and mothers of children with Down syndrome had 1.34 more years of education, on

\begin{tabular}{llccc}
\hline & \multicolumn{2}{c}{ Down syndrome } & \multicolumn{2}{c}{ Mixed etiologies } \\
& Mor \% & SD & Mor \% & SD \\
\hline Child gender (\% Male) & $66.7 \%$ & & $48.5 \%$ & \\
Ethnicity & & & & \\
European American & $87.5 \%$ & & $84.8 \%$ & \\
Asian/Pacific Islander & $4.2 \%$ & & $3.0 \%$ & \\
African American & $4.0 \%$ & & $6.1 \%$ & \\
Other & $8.3 \%$ & & $6.1 \%$ & \\
Family income (Time I) & 52,708 & 37,459 & $33,28 \mathrm{I}$ & 260 \\
Mothers' age (years) & 34.42 & 4.53 & 30.73 & 5.51 \\
Mothers' education (years) & 14.79 & 1.93 & 13.45 & .50 \\
Married (\%) & $88.0 \%$ & & $81.6 \%$ & \\
\hline
\end{tabular}

Table I. Demographic information by Diagnostic Group 
average. Because of these demographic differences, family income, maternal education, and maternal age were considered in all subsequent analyses.

Children in both groups were equated on Bayley mental scale raw scores at Time $1, t(55)=.07, p=.94$, (Down syndrome $M=47.33, S D=8.62$; Mixed $M=47.52, S D=11.62$; see Table 1). As per Mervis and Klein-Tasman (2004), raw scores were used for these analyses because a large percentage of children in both groups performed at the floor (score $=49$ ) of the available standard scores for the Bayley. Our matching procedures, which involved collected data at the same point in time for each child, made it possible to compare across groups on raw scores. In addition, raw scores have been used in a large number of studies of cognition in Down syndrome (Byrne, McDonald, \& Buckley, 2002; Boudreau, 2002; Cupples, \& Iacano, 2000; Fidler, Most \& Guiberson, in press; Kay-Raining Bird, Cleave, \& McConnell, 2000; Laws, \& Gunn, 2002).

\section{Procedure}

Families were recruited from 17 participating clinics or agencies providing early intervention services for infants with diagnosed disabilities, or follow-up services for infants at biomedical risk. A staff member at each agency identified infants who were the appropriate age, and then gave these infants' mothers a descriptive pamphlet about the study and asked if a member of our research team could telephone them to describe the study in more detail. Our project staff obtained the names and telephone numbers of mothers who agreed to be called, and they contacted and recruited the families by phone (A few families contacted us after hearing about the study from a friend, or obtaining information from a poster about the study). The staff did not recruit mothers of infants with significant sensory impairments, due to assessment difficulties.

At 12 months of age, an evaluator conducted a home visit to enrol the family into the study, to collect initial data about the family and child, and to assess the child's development. At 30 and 45 months of age, children visited the laboratory playroom to assess child developmental outcomes and collect parenting stress information. For all child assessments, scores were adjusted for prematurity.

\section{Measures}

1. Demographics Questionnaire. Mothers provided information at the 12-month visit (and updated it at 30 and 45 months) regarding the study child's birth order, gender, and ethnicity; the mother's age, education, and marital status; and family income. We calculated the family income-to-needs ratio by dividing family income by the poverty threshold (from U.S. Census Bureau tables) for the year of each interview.

2. Bayley Scales of Infant Development. A trained evaluator administered the revised Bayley Scales of Infant Development (BSID-II; Bayley, 1993) to the study children at 12 months and 30 months, yielding scores on the Mental Scale, the Motor Scale, and the Behaviour
Rating Scale (BRS) for each time point. For the purposes of this study, only the Mental Scale was included to match the two groups for severity of impairment.

The Bayley scales are the most widely used assessments of developmental progress in children at biomedical risk or with disabilities. They were designed originally to assess typically developing infants. However, the revised version was standardised on a broader range of children, including those born prematurely, and those who have Down syndrome, developmental delay, or autism. The evaluator adapted the Bayley assessment procedures to the special needs of the children in the study, if necessary, without compromising the validity of the test (e.g., using an adaptive device to maintain the child in an upright position during mental testing).

3. Infant Temperament Questionnaire-Revised. Mothers completed the Revised Infant Temperament Questionnaire (ITQ; Carey \& McDevitt, 1978) to provide an evaluation of their child's difficult temperament at 12 months. The 95-item ITQ was reduced to 39 items for the large scale NICHD Study of Early Child Care, and this version was used in the present report. The mothers rated each item on a 6-point Likert-type scale.

4. Child Behaviour Checklist (Achenbach, 1991a). At Time 2 and Time 3 (30 and 45 months), mothers completed the 99-item Child Behaviour Checklist (CBCL; Achenbach, Edelbrock, \& Howell, 1987) to assess the children's behaviour problems. This CBCL is a widelyused, nationally normed measure that has also been demonstrated to be appropriate for young children with special needs (Dykens \& Kasari, 1997; Shonkoff \& Upshur, 1993). The CBCL has been standardised on large, representative samples of children from across the United States. Age and sex normed standard (T) scores for behaviour problems, with cut-off scores for clinically significant problems (above the 98th percentile), are provided.

The CBCL yields broad-band factors of internalising and externalising problems, as well as narrow-band factors assessing social withdrawal, aggression, anxiety, and overactivity. For the purposes of this study, we explore only the broad-band factors. The test-retest reliability, internal consistency, concurrent and predictive validity, and the construct validity of these scales have been documented in hundreds of studies in North America and in other cultures (see Achenbach, 1991a; Achenbach, 1991b; Achenbach \& Brown, 1991). For example, one-week test-retest reliability on the parent version, obtained on a large sample of 4-16 year olds, averaged $\mathrm{r}=.89$; inter-parent agreement averaged $r=.70$; stability of scale scores over a two-year follow-up period had a mean $r=.71$. Narrow- and broad-band scores also clearly differentiate referred from non-referred children (Achenbach, 1991a). The externalising, internalising, and total T-scores were used in the present report. 


\section{Analyses}

This study focuses on exploring a variety of associations that may inform early intervention science and practice in Down syndrome. As such, the analytic emphasis is on estimating quantities of interest (e.g., between-group differences, regression slopes) rather than on conducting dichotomousoutcome null-hypothesis significance tests. Consistent with the recommendations generated by the American Psychological Association Task Force on Statistical Inference (Wilkinson et al., 1999), unstandardised point and interval estimates are the primary results presented here instead of $\mathrm{p}$-values. While interval estimates can be interpreted in a significance testing fashion (i.e., an interval either does or does not contain a point null), they have the important advantage of offering a full range of plausible values for the parameter of interest. An interval estimate as a whole also conveys the degree of precision with which a parameter has been estimated. In sum, it is of interest here to address the "how much" questions (e.g., how much is the difference?) rather than the less informative dichotomous-response "is there" questions (e.g., is there a difference?).

\section{Results}

\section{Temperament/behaviour problems at 12 , 30 , and 45 months}

Analyses were conducted to examine the extent to which the groups differed from one another in their temperament ratings at Time 1, and their CBCL ratings at Times 2 and 3 . Within-group means and standard deviations for these variables are presented in Table 2. Results (test statistics and p-values) of tests of the null hypothesis that the population mean are equal between groups at each time point are also presented in Table 2. At Time 1, group means differed by approximately two points on the Infant Temperament Questionnaire. A 95\% confidence interval for this difference is $(-7.4,11.3)$. At Time 2 , the mean Total Problem Behaviour $\mathrm{T}$ score was approximately 4.6 points lower for the Down syndrome than for the mixed comparison group. Further exploration suggests that this difference is a function of lower Internalising Behaviour T scores in the Down syndrome group. A 95\% confidence interval for the between-group difference in mean Internalising Behaviour T scores ranges from .2 to 11.6. It is also important to note that there was significantly less variability along this dimension in the Down syndrome group than there was in the mixed comparison group, Levene's $F=4.66, p<.05$.

In contrast, a between-group difference of only 1.8 points was observed for the mean Externalising Behaviour T score. A 95\% confidence interval for this difference is $(-3.0,6.7)$. At Time 3, no substantively meaningful between-group differences (see Table 2) were observed for any of the CBCL broad band measures (Externalising Behaviour, Internalising Behaviour, and Total Problem Behaviour).

\section{Changes in maladaptive behaviour from Time 2 to Time 3}

Time 2 to Time 3 individual difference scores were computed for all of the CBCL broad band measures. As elucidated by Rogosa (1988), a difference (or change) score is a reliable and useful measure of the magnitude of a change in score over time as long as there are some differences across individuals in change (i.e., not every individual experiences the same amount and direction of change). A mean increase of approximately 3.6 points in the Total Problem Behaviour $\mathrm{T}$ score was observed for the Down syndrome group while a mean decrease of 1.5 points was observed for the mixed comparison group. Further exploration suggested that this between-group difference is driven primarily by a mean increase of 5.3 points in Internalising Behaviour $\mathrm{T}$ scores in the Down syndrome group from Time 2 to Time 3. A mean decrease of only .6 points was observed for the 
mixed comparison group. A 95\% confidence interval for the between-group difference in mean Internalising Behaviour $\mathrm{T}$ change scores is $(1.5,10.4)$. In contrast, a between-group difference in mean change scores of only 2.0 points was observed for the Externalising Behaviour T scores. A 95\% confidence interval for this difference is $(-2.4,6.4)$.

In order to explore the possibility of effects of betweengroup demographic differences on all of the findings above, prediction equations were estimated for temperament ratings at Time 1, CBCL ratings at Times 2 and 3, and the Time 2 to Time 3 CBCL change scores. In all cases, once group was taken into account, inclusion of demographic variables did not significantly improve prediction of temperament or CBCL or meaningfully reduce the observed standard error of the estimate. Therefore, while there are some demographic differences between groups, these differences do not alter interpretation of the results described thus far.

\section{Temperament and maladaptive behaviour at Time 3}

The longitudinal nature of these data was also of interest in this study. In order to explore this association, maladaptive behaviour at Time 3 was regressed on infant temperament within each group. The results (see Table 3 ) indicate that differences in temperament are associated with differences in maladaptive behaviour at Time 3 that are, on average, more than four times larger in the mixed comparison group than in the Down syndrome group (i.e., the point estimates and 95\% confidence intervals for the mixed comparison group and the Down syndrome group were $.33(.08, .59)$ and .07 $(-.21, .36)$, respectively). While the confidence intervals for each slope are rather wide due to the small sample size, the data suggest that higher infant temperament scores are associated with "higher" maladaptive behaviour at Time 3 in the population of children with developmental disabilities of mixed etiologies. In addition, while approximately $24 \%$ of the variability in maladaptive behaviour at Time 3 can be accounted for by infant temperament in the mixed comparison group, only $2 \%$ of the variability can be accounted for in the Down syndrome group. In sum, infant temperament may be a better predictor of maladaptive behaviour at Time 3 in the mixed comparison group than in the Down syndrome group.

\section{Discussion}

Though individuals with Down syndrome tend to show comparatively lower levels of psychopathology than other individuals with developmental disabilities, they do show increased risk for certain types of behaviour problems compared with children without developmental disabilities.

\section{Comparison Group}

\section{Down Syndrome Group}

This study is among the first to explore the early emergence of behavioural issues in young children with Down syndrome. Temperament was assessed at 12 months, and the association between early temperament and behavioural problems at 30 and 45 months was explored. As such, this study offers an important contribution to the growing study of the emergence of the Down syndrome behavioural phenotype (Fidler, 2005).

Several findings from this study are notable. First, at Time 1 and Time 3, no substantively meaningful differences in temperament or behaviour problems were observed between young children with Down syndrome and the mixed group. Specifically, no Down syndrome group mean was more than two percent more or less than the comparable mixed group mean for any measure. This suggests that the findings previously reported of lower maladaptive behaviour and psychopathology in children with Down syndrome relative to children with other developmental disabilities are not consistent with this study's measurements at 12 months and 45 months. At Time 2, however, young children with Down syndrome showed lower Total CBCL scores than did children in the mixed comparison group. Closer examination of CBCL profile suggests that lower levels $(\sim 17 \%)$ of internalising scores are driving this difference at Time 2 .

By Time 3, children in the Down syndrome group showed increases in internalising behaviour such that their scores were no longer lower than scores observed in the mixed group. The changes in internalising T-scores were much greater in the Down syndrome group than in the mixed comparison group from Time 2 to Time 3 . This suggests that the onset of Internalising Behaviour difficulties in young children with Down syndrome may emerge later in early development than in children with developmental disabilities of mixed/nonspecific etiologies.

In addition to describing between-group differences at each time point, the association between temperament and outcomes at Time 3 also was explored within each group. Findings from this part of the study suggest that temperament at Time $\mathrm{l}$ is a much stronger predictor of maladaptive behaviour outcomes at Time 3 in the mixed comparison group than in the Down syndrome group. Thus, while

\begin{tabular}{|l|l|l|}
\hline Predictor & Unstandardised Coefficient (SE) & $\mathbf{9 5 \%}$ Confidence Interval for Slope \\
\hline Infant Temperament & $.33(.12)^{*}$ & $(.08, .59)$ \\
$\mathrm{R}^{2}$ & .24 & \\
\hline
\end{tabular}

\begin{tabular}{|l|l|l|}
\hline Predictor & Unstandardised Coefficient (SE) & 95\% Confidence Interval for Slope \\
\hline Infant Temperament & $.07(.14)$ & $(-.21, .36)$ \\
$\mathrm{R}^{2}$ & .13 & \\
\hline
\end{tabular}

* Coefficient is more than twice its standard error

Table 3. Regression of maladaptive behaviour at Time 3 on infant temperament by group 
children in the mixed group show a discernable pattern of behavioural development during the first 3 years, it is quite plausible that, for children with Down syndrome, knowing early temperament is uninformative with respect to later maladaptive behaviour.

This finding of a uniquely unpredictable trajectory of the early development of maladaptive behaviour in Down syndrome has important implications for intervention for young children with Down syndrome. Anecdotally, parents of toddlers with Down syndrome describe shifts in behavioural style wherein a previously placid, easy temperament infant becomes a toddler posing new behavioural difficulties for their parents (sudden changes in mood, withdrawing from situations). While increases in behavioural challenges in toddlerhood are typical, the changes in $\mathrm{T}$ scores observed in this study suggest that young children with Down syndrome may make a more pronounced shift in the development of internalising behaviours than do typically developing children and children with other developmental disabilities. While findings from this study suggest that infants in the comparison group who show easier temperaments are likely to be children with lower levels of behavioural problems in toddlerhood, data in the Down syndrome group suggest that easier temperaments in infancy do not predict lower levels of maladaptive behaviour. Thus, parents of children with Down syndrome may experience a shift in their child's behaviour that is more pronounced than other parents experience, which may necessitate increased parenting supports to understand and strategise for their child.

There are several important limitations to this study that must be considered. First, because of the developmental nature of this study - where children were assessed at 12 , 30 , and 45 months - it was not possible to include identical measures at all time points. Maladaptive behaviour as a construct only emerges in toddlerhood, and was not quantifiable at Time 1. The construct of temperament was measured in its place. However, because of the construct changed from the first to second timepoints, it was impossible to describe growth trajectories across more than the final two timepoints. In addition, this study was conducted with relatively small samples of children in each diagnostic group. As such, it warrants replication with larger sample sizes.

It is also important to note that the two diagnostic groups differed demographically from one another in important ways from the outset. Specifically, mothers of children with Down syndrome were approximately 4 years older than mothers of children in the mixed comparison group, and median family income was approximately $\$ 20,000$ higher in the Down syndrome group. This pattern of differences is not uncommon in studies where families of children with Down syndrome are compared with families of children with other developmental disabilities (Cahill \& Glidden, 1997). In all cases, however, once diagnostic group was taken into account, inclusion of demographic variables did not significantly improve prediction of temperament or
CBCL or meaningfully reduce the observed standard error of the estimate.

Thus, compared with other children with developmental disabilities, young children with Down syndrome show lower levels of internalising behaviour at 30 months. However, their rates of internalising behaviour seem to increase greatly by 45 months. Furthermore, 12 -month temperament ratings in children with Down syndrome were not predictive of later maladaptive behaviour outcomes. The time-sensitive nature of this profile may interact with the emerging phenotype to contribute to the personality-motivation orientation described in this population (Fidler, in press), and may warrant targeted and time-sensitive intervention.

\section{Acknowledgements}

This study was supported by grant MCJ-530640 (Cathryn Booth-LaForce, PI) from the Maternal and Child Health Bureau (Title V, Social Security Act), Health Resources and Services Administration, Department of Health and Human Services. We thank Jacqueline Bacus, Darlene Todd, Jennifer Page, Karen Halsey, Lynne Cochran, Jennifer Duval, and Donald Goldstein for their assistance. We also thank the families who participated in this study.

\section{Correspondence}

Deborah J. Fidler, Ph.D. • 102 Gifford Building, 502 West Lake Street, Colorado State University, Fort Collins, CO, 80523・E-mail: Deborah.Fidler@colostate.edu.

\section{References}

Achenbach, T. M. (1991a). Manual for the Child Behaviour Checklist and the Revised Child Behaviour Profile. (2nd $e d$.$) . Burlington: University of Vermont.$

Achenbach, T. M. (1991b). Manual for the Teacher's Report Form and 1991 Profile. Burlington, VT: University of Vermont.

Achenbach, T. M. \& Brown, J. S. (1991). Bibliography of published studies using the Child Behaviour Checklist and related materials: 1991 edition. Burlington, VT: University of Vermont Department of Psychiatry.

Achenbach, T., Edelbrock, C. \& Howell, C.T. (1987). Empirically based assessment of the behavioral/emotional problems of 2- and 3-year old children. Journal of Abnormal Child Psychology, 15, 629-650.

Bayley, N. (1993). Bayley Scales of Infant Development: Second Edition. San Antonio, TX: Psychological Corporation.

Boudreau, D. (2002). Literacy skills in children and adolescents with Down syndrome. Reading and Writing: An Interdisciplinary Journal, 15, 497-525.

Byrne, A., Buckley, S., MacDonald, J. \& Bird, G. (1995). Investigating the literacy, language, and memory skills of children with Down's syndrome. Down's syndrome: Research and Practice, 3, 53-58.

Cahill, B.M. \& Glidden, L.M. (1997). Influence of child diagnosis on family and parental functioning: Down syndrome versus other disabilities. American Journal on Mental Retardation, 101, 149-160. 
Carey, W.B. \& McDevitt, S.C. (1978). Revised Infant Temperament Questionnaire. Pediatrics, 61, 735-739.

Collacott, R.A., Cooper, S. \& McGrother, C. (1992). Differential rates of psychiatric disorders in adults with Down's syndrome compared with other mentally handicapped adults. British Journal of Psychiatry, 161, 671-674.

Cupples, L. \& Iacono, T. (2000). Phonological awareness and oral reading skills in children with Down syndrome. Journal of Speech, Language, and Hearing Research, 43, 595-608.

Dykens, E.M. \& Hodapp, R.M. (2001). Research in mental retardation: Toward an etiologic approach. Journal of Child Psychology and Psychiatry and Allied Disciplines, 42, 49-71.

Dykens, E.M. \& Kasari, C. (1997). Maladaptive behaviour in children with Prader-Willi syndrome, Down syndrome, and non-specific mental retardation. American Journal on Mental Retardation, 98, 580-587.

Dykens, E., Shah, B., Beck, T. \& King, B.H. (2002). Maladaptive behaviour in children and adolescents with Down's syndrome. Journal of Intellectual Disability Research, 46, 484-492.

Fidler, D.J. (2005). The emerging Down syndrome behavioral phenotype in early childhood: Implications for practice. Infants and Young Children, 18, 86-103.

Fidler, D.J. (in press). The emergence of a syndrome-specific personality-motivation profile in young children with Down syndrome. In J.A. Rondal (Ed.) \& J. Perera (Ed). Down syndrome: Neurobehavioural Specificity. Whurr Publishers.

Fidler, D.J., Most, D.E. \& Guiberson, M.M. (2005). Neuropsychological correlates of word identification in Down syndrome. Research in Developmental Disabilities, 26, 487-501.

Kay-Raining Bird, E., Cleave, P.L. \& McConnell, L. (2000). Reading and phonological awareness in children with Down syndrome. American Journal of Speech-Language Pathology, 9, 319-330.

Kent, L., Evans, J., Paul, M. \& Sharp, M. (1999). Comorbidity of autistic spectrum disorders in children with Down syndrome. Developmental Medicine and Child Neurology, 41, 153-158.

Laws, G. \& Gunn, D. (2002). Relationships between reading, phonological skills and language development in individuals with Down syndrome: A five year follow-up study. Reading and Writing, 15, 527-548.

Lund, J. (1985). Psychiatric aspects of Down's syndrome. Acta Psychiatrica Scandinavia, 78, 369-374.

Mervis, C. \& Klein-Tasman, B. (2004). Methodological issues in group-matching designs: $\alpha$ levels for control variable comparisons and measurement characteristics of control and target variables. Journal of Autism and Developmental Disorders, 34(1), 7-17.

Myers, B.A. \& Pueschel, S.M. (1991). Psychiatric disorders in persons with Down syndrome. Journal of Nervous and Mental Disease, 179, 609-613.

Paterson, S.J., Brown, J.J., Gsoedl, M.K., Johnson, M.H. \& Karmiloff-Smith, A. (1999). Cognitive modularity and genetic disorders. Science, 286, 2355-2358.

Robins, L. (1966). Deviant children grown up. Baltimore: Williams and Wilkins.
Robins, L. (1978). Sturdy childhood predictors of adult antisocial behavior: Replications from longitudinal studies. Psychological Medicine, 8, 611-622.

Robins, L. \& Rutter, M. (1990). Straight and devious pathways from childhood to adulthood. Cambridge, England: Cambridge University press.

Rogosa, D. (1988). Myths about longitudinal research. In Schaie, K., Cambell, R., Meredith, W. \& Rawlings, S. (Eds.), Methodological issues in aging research. New York: Springer.

Shonkoff, J. P. \& Upshur, C. C. (1993, March). Changes in the impact of the caregiving environment on emerging child competencies. Paper presented at the meeting of the Society for Research in Child Development, New Orleans, LA.

Stores, R., Stores, G., Fellows, B. \& Buckley, S. (1998). Daytime behaviour problems and maternal stress in children with Down's syndrome, their siblings, and non-intellectually disabled and other intellectually disabled peers. Journal of Intellectual Disability Research, 42, 228-237.

Wilkinson, L. \& the Task Force on Statistical Inference. (1999). Statistical methods in psychology journals: Guidelines and explanations. American Psychologist, 54, 594-604. 